\title{
ALPHA POWER CHANNELLING WITH TWO WAVES
}

\author{
N.J. FISCH, M.C. HERRMANN \\ Princeton Plasma Physics Laboratory, \\ Princeton University, \\ Princeton, New Jersey, \\ United States of America
}

\begin{abstract}
The complete channelling of energy from $\alpha$ particles is likely to be realized only through the excitation of a variety of waves, rather than by one wave alone. While one wave constrains more firmly the direction of the energy transfer, the necessary wave characteristics are far more easily achieved through a combination of waves, even at the expense of less restrictive motion of the $\alpha$ particles.
\end{abstract}

\section{INTRODUCTION}

Fusion reactors burning deuterium and tritium (DT) fuel are sustained by $\alpha$ particle heating. Generally, the $\alpha$ particles heat mainly electrons through Coulomb collisions. However, in the presence of appropriate waves, the $\alpha$ particles might amplify these waves on a collisionless time scale. If the waves then are absorbed either by electrons travelling in one direction to accomplish current drive [1] or are absorbed by fuel ions for increased reactivity [2], the $\alpha$ particle power has been effectively 'channelled' to accomplish more useful functions than mere electron heating. The amplification of the waves can occur if there is a population inversion in the $\alpha$ particles along the wave diffusion path, where the diffusion path points from high energy $\alpha$ particles in the plasma centre to low energy $\alpha$ particles at the plasma periphery. Complete channelling of the $\alpha$ particle power to ions would result in about a factor of two in the fusion power density, where most of this increase is due to the operation in the so-called 'hot ion' mode $[3,4]$.

Although, in principle, substantial channelling of $\alpha$ particle energy can occur, exactly how to accomplish this effect is a challenging problem. Wave interactions that tend to drive particles in velocity space only tend not to extract most of the recoverable energy [5-7]. A poloidally and toroidally propagating lower hybrid wave was proposed [1] to make use of the free energy available in diffusing $\alpha$ particles also in space, but this wave alone appears to be difficult to excite with the required wave characteristics $[8,9]$. One of the more promising wave candidates is the short wavelength mode converted ion Bernstein wave $[10,11]$. However, this wave is hard to excite with sufficient toroidal wavenumber to deliver $\alpha$ particles to the plasma periphery.
This work shows that qualitatively different behaviour occurs when $\alpha$ particles are resonant with more than one kind of wave. In particular, we show that waves in the ion cyclotron range of frequencies, such as the ion Bernstein wave (IBW), may be combined to good effect with low frequency waves, such as the toroidal Alfvén eigenmode (TAE). For certain $\alpha$ particles, it can be seen that the presence of either of these waves alone leads to almost no energy extracted, while almost complete energy extraction can occur when both waves are present. The extent to which substantially all the $\alpha$ particles can be controlled in a similar fashion will determine the utility of using such waves.

The paper is organized as follows: In Section 2, we review the issues in energy extraction by one wave. In Section 3, we show how qualitatively different behaviour can occur with more than one wave. In Section 4, we show as an example how an IBW and a TAE-like wave can possess complementary functions. In Section 5, we discuss the limitations and implications of this work.

\section{CONSTRAINING DIFFUSION TO ONE DIMENSION}

The energy of the $\alpha$ particles is best tapped by diffusing them in energy and space. The channelling occurs when the diffusion path connects high energy $\alpha$ particles at the plasma centre to low energy $\alpha$ particles at the plasma periphery. When one wave is utilized, there can be only one diffusion path, so that there are stringent constraints on the $\alpha$ particle motion. If the path is chosen appropriately, then, if an $\alpha$ particle gains energy, it must diffuse to the tokamak periphery; conversely, if it were to lose energy in interacting 
with the wave, it must diffuse to the tokamak centre. The $\alpha$ particle motion is constrained to lie on a one dimensional curve, a line. Thus, particles diffused by the wave to the tokamak periphery give up a precise amount of energy to the wave which is proportional to their distance travelled in reaching the periphery.

For simplicity in explaining the effect, we consider a slab geometry, where we can write the ratio of displacement of the guiding centre in the $x$ direction, $\Delta x_{\mathrm{gc}}$, to energy absorbed as

$$
\Delta x_{\mathrm{gc}}=-k_{y} \Delta \epsilon / m \Omega \omega
$$

This quantity is determined by wave and particle parameters only; $\omega$ is the wave frequency, $k_{y}$ is the wavenumber in the $y$ direction, $m$ is the $\alpha$ particle mass and $\Omega \equiv q B / m$ is the $\alpha$ particle gyrofrequency. In the slab case, upon repeated interactions with the wave, a particle will trace a line in $\epsilon-x_{\mathrm{gc}}$ space.

Suppose that the plasma boundary is at $x=a$, i.e. $\alpha$ particles can only leave at $x=a$. The plasma centre is at $x=0$, by which is meant that no $\alpha$ particles can leave at $x=0$. For efficient channelling, one would then require $\Delta x_{\mathrm{gc}} / \Delta \epsilon \sim a / \epsilon_{\alpha}$, where $a$ is the extent of the plasma and $\epsilon_{\alpha}$ is the $\alpha$ particle birth energy. If, instead, $\Delta x_{\mathrm{gc}} / \Delta \epsilon \gg a / \epsilon_{\alpha}$, then the $\alpha$ particle would be extracted from the centre with almost all its energy intact whereas, if $\Delta x_{\mathrm{gc}} / \Delta \epsilon \ll a / \epsilon_{\alpha}$, then the $\alpha$ particles are not extractable from the plasma centre. In this case, a population inversion is not likely to occur, and the wave will not be amplified.

In toroidal geometry, particles interacting with one wave trace a line in $\epsilon-\mu-P_{\phi}$ space, where $\mu=m v_{\perp}^{2} / 2 B$ is the magnetic moment, $\epsilon=\mu B+m v_{\|}^{2} / 2$ is the kinetic energy, $P_{\phi}=R\left(m B_{\phi} v_{\|} / B+q A_{\phi}\right)$ is the canonical angular momentum and $A_{\phi}$ is the vector potential. Each point in $\epsilon-\mu-P_{\phi}$ space represents a single guiding centre orbit for trapped particles, and, for each sign of $v_{\|}$, a passing particle orbit. Given $\epsilon, \mu$ and $P_{\phi}$, and the sign of $v_{\|}$for passing orbits, it may be determined if the orbit intersects the plasma periphery, thus losing the particle $[12,13]$. Particles tend to follow closely the magnetic surfaces, i.e. surfaces of constant $R A_{\phi}$, so that the particle position is largely determined by $P_{\phi}$, especially for low energy particles.

Upon interaction with a wave with toroidal mode number $n_{\phi}$, and absorbing energy $\Delta \epsilon, P_{\phi}$ changes by

$\Delta P_{\phi}=\left(n_{\phi} / \omega\right) \Delta \epsilon$

Assume that the exchange of energy occurs only for particles satisfying the resonance condition

$\omega-k_{\|} v_{\|}=n \Omega$ where $n$ is an integer, then, upon absorbing energy $\Delta \epsilon$, $\mu$ changes by

$\Delta \mu=(n Z e / m \omega) \Delta \epsilon$

where $e$ is the charge on an electron and, for $\alpha$ particles, $Z=2$. Thus, upon repeated interactions with one wave, the constants of motion, $\epsilon, \mu$ and $P_{\phi}$, trace a line.

Waves with the appropriate characteristics must be excited both to extract energy and to deliver the $\alpha$ particle to the periphery. A particularly promising wave in a tokamak is the mode converted IBW wave, excited with high $k_{\theta}$, where $\theta$ is the poloidal mode number [10]. This wave breaks the $\mu$ invariance of the $\alpha$ particle motion, but its excursion is limited, by Eq. (2), if $n_{\phi}$ is not sufficiently large. In Fig. 1, we show the orbit of an $\alpha$ particle interacting with an IBW wave with $n_{\phi}=50$. The outer orbit is the largest orbit the particle can sample and still be resonant with the wave. In this orbit, the $\alpha$ particle has lost about $2 / 3$ of its initial energy and essentially all of its perpendicular energy. At this point, constrained through Eq. (4), the $\alpha$ particle can lose no more energy and so cannot quite make it to the periphery. The magnetic geometry for this case is TFTR-like, with concentric circular flux surfaces, $2 \mathrm{MA}$ toroidal current and $5.3 \mathrm{~T}$

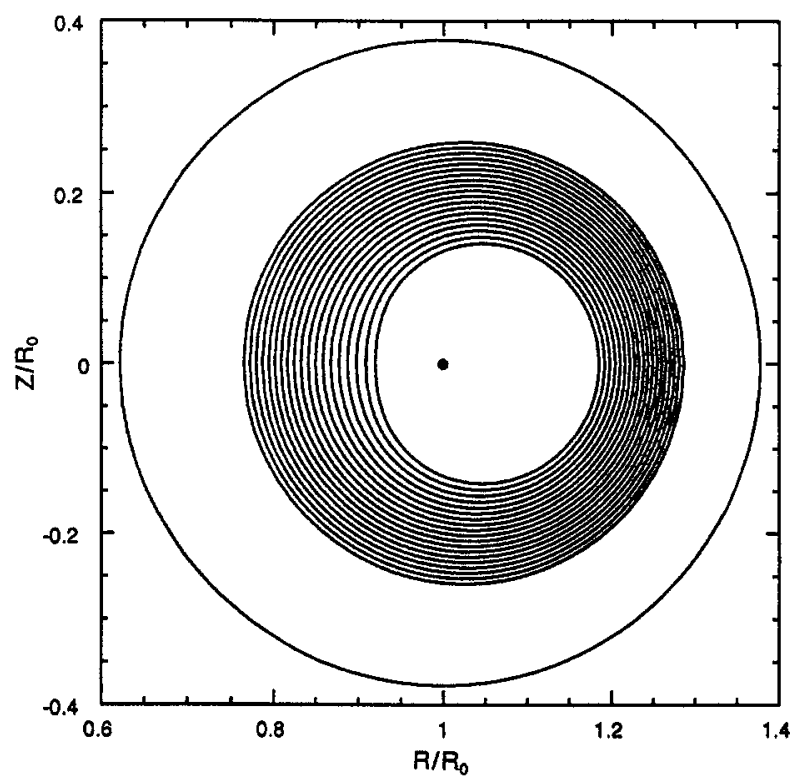

FIG. 1. Poloidal cross-section of orbit of a co-going $\alpha$ particle interacting solely with a mode converted ion Bernstein wave $(M C I B W)$. Magnetic axis is at $(1,0)$. If the particle were to lose energy under interaction with this wave it would move from the central orbit to the outermost orbit, eventually losing $2.5 \mathrm{MeV}$. 
toroidal field. The major radius at the magnetic axis is $R_{0}=2.6 \mathrm{~m}$, and the minor radius is $a=1 \mathrm{~m}$. The mode converted wave, at $32 \mathrm{MHz}$, exists near the mode conversion layer in a vertical slab between $1.05 R / R_{0}$ and $1.15 R / R_{0}$. The vertical extent of the slab is the full tokamak minor cross-section, while the horizontal extent is $26 \mathrm{~cm}$. The $\alpha$ particle could be extracted either in a lower current equilibrium or in the presence of a higher mode number IBW wave. However, in practice, higher mode numbers in a TFTR-size tokamak would be difficult to excite efficiently.

\section{DIFFUSION ALONG A TWO DIMENSIONAL SURFACE}

There are a number of obstacles in channelling $\alpha$ particle power with just one wave. First, the necessary wave characteristics are difficult to obtain. In the case of Fig. 1, these high- $n_{\phi}$ modes are not easily excited in a tokamak. Second, even if such a wave could be excited, it tends mainly to extract the perpendicular energy only of the $\alpha$ particle. Third, there is the possibility of 'hard landings' [11]; if the $\alpha$ particle were born away from the plasma centre, it would have a shorter distance to travel to the periphery, and, hence, it would leave with much of its energy intact.

A qualitatively different picture emerges when more than one wave interacts with the $\alpha$ particle. Consider, again for simplicity, a slab geometry. If the waves induce different ratios of $\Delta x_{\mathrm{gc}} / \Delta \epsilon$, the $\alpha$ particles are no longer constrained to a line in $x-\epsilon$ space. If an $\alpha$ particle encounters the periphery, its energy is no longer immediately determined from its initial co-ordinates; its energy at the wall would depend on the history of how it received kicks from each of the waves with which it was resonant.

However, by interacting with more than one wave, the constraints on each of the waves for expelling the $\alpha$ particle are relaxed. In addition, depending on the waves involved, it may be that, even though the $\alpha$ particle is not constrained to lose energy upon expulsion from the plasma, the $\alpha$ particle may still be vastly predisposed to lose energy to the waves rather than to gain energy. To illustrate this, consider the interaction of $\alpha$ particles with two waves in a slab, as sketched in Fig. 2. Here, $x=0$ depicts the plasma centre, while the plasma periphery is at $x=a$. Alpha particles can leave the plasma at $x=a$ only. In Fig. 2(a), we depict by horizontal lines diffusion paths in energy only, such as might result from a Landau-type resonance. These diffusion paths can have finite extent in the horizontal direction, and these line segments, of course, need not be the same length, since the resonance condition may be energy and space dependent. By diffusing along these paths only, $\alpha$ particles born near the plasma centre cannot reach the plasma boundary, as all $\alpha$ particles are effectively confined to horizontal line segments in $x-\epsilon$ space. Similarly, we depict, by vertical line segments, diffusion paths in space only, such as might result from interactions with a magnetic ripple. By diffusing along these paths only, $\alpha$ particles born near the plasma centre reach the plasma boundary, but without any loss in energy.

Clearly, in the presence of either resonance alone, $\alpha$ particles sample only line segments, whereas in the presence of both resonances at once, $\alpha$ particles can sample a two dimensional (2-D) volume in $x-\epsilon$ space. (a)

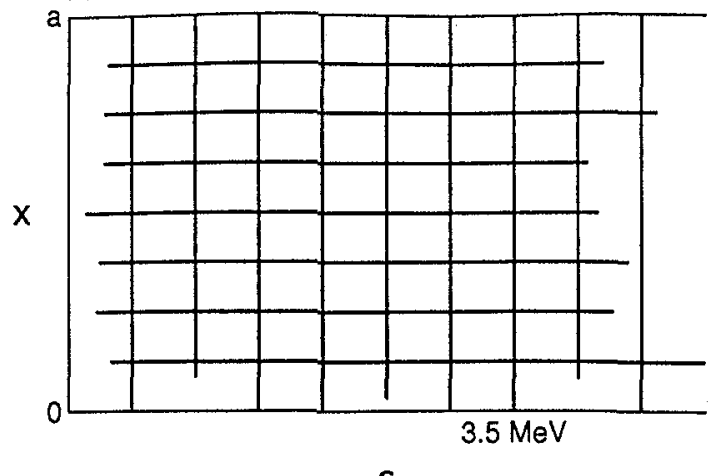

(b)

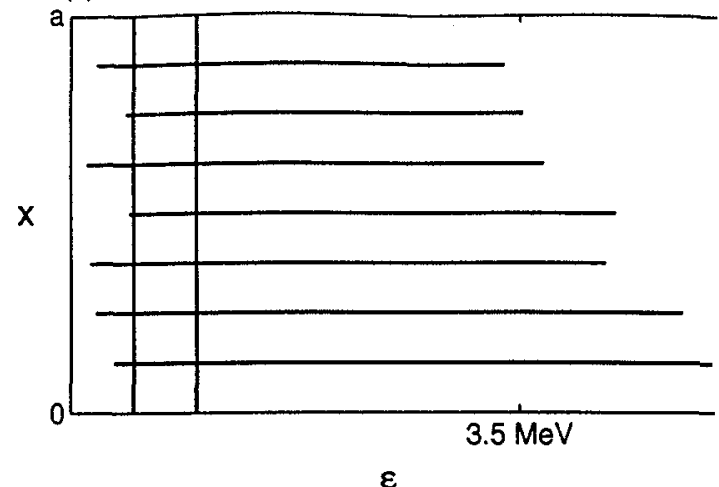

$\varepsilon$

FIG. 2. (a) Schematic diffusion paths in energy and space. Diffusion in energy $\epsilon$ is exhibited by horizontal paths; diffusion in space $\mathrm{x}$ by vertical paths. The plasma periphery is at $\mathrm{x}=\mathrm{a}$; the plasma centre is at $\mathrm{x}=0$. (b) Schematic diffusion paths in energy and space. The diffusion in space has a strong energy dependence. 
In the presence of just one wave, the $\alpha$ particle may not be able to access the plasma boundary, but if it does come into contact with the boundary, it is constrained to do so at one precise energy. On the other hand, when diffusion along multiple diffusion paths occurs, it is apparent that $\alpha$ particles leaving the plasma are not constrained to do so at a single energy.

There are, however, a number of ways in which control can be exercised in the presence of multiple waves. Let us imagine particles diffusing along multiple diffusion paths as receiving random kicks in a 2-D web. Strands within the web may be removed or strengthened by means of resonance conditions. Thus, although the kicks are random, structure in the web or surface itself can lead to diffusion in a definite direction. As an example, consider Fig. 2(b), where we imagine that diffusion in energy is of finite extent, whereas the diffusion in space is energy dependent, say, with significant diffusion only at low energy. Such an energy dependent diffusion in space only has, in fact, been investigated $[14,15]$. Clearly, all $\alpha$ particles must leave the plasma at low energy, so that there is net extraction of the $\alpha$ particle energy.

In toroidal geometry, the details are somewhat more complicated, but the idea is the same - namely, one wave diffuses along a line, while several waves diffuse along a web in $\epsilon-\mu-P_{\phi}$ space. Again, structure in the web can lead to usefully constrained motion of the particles.

\section{DIFFUSION BY TWO WAVES IN A TORUS}

In optimizing energy extraction through diffusion by two waves, one strategy would be to have the two waves play complementary roles. For example, choose one wave in the ion cyclotron range of frequencies in order to break the invariance of $\mu$ and thereby access the particle's perpendicular energy. The mode converted IBW [16] could play this role admirably. What the IBW does not do easily is to move the a particles large distances. The second wave then need not break the $\mu$ invariance, but ought to move the $\alpha$ particles large distances with little energy exchange. Low frequency modes, such as the TAE, that do not break the adiabatic motion of the $\alpha$ particles, should serve well the complementary role.

The IBW wave may be excited by launching a fast wave that mode converts at the ion-ion hybrid resonance layer [17], or through other mode conversion techniques [18]. This wave has been excited with toroidal phasing in TFTR in order to drive plasma current [19]. In addition, it appears to interact with fusion by-products [20]. The TAE mode [21, 22] has been extensively analysed theoretically, including models for its excitation and saturation [23-25]. It has been predicted to interact effectively with $\alpha$ particles, expelling them with little energy loss [26]. This mode has been driven directly in JET using a saddle coil antenna [27].

In this section, we demonstrate the qualitatively different motion that results when such waves are used in combination. In order to demonstrate this difference we employ highly simplified models that capture the essential features of both the IBW wave and a TAElike wave in a TFTR-size tokamak. The model that we use is that if a particle is resonant with a wave at any point in its orbit, i.e. if it satisfies locally Eq. (3), then it experiences a jump to a nearby orbit, or point in $\epsilon-\mu-P_{\phi}$ space. The nearby point meets the criteria, $\epsilon \rightarrow \epsilon+\Delta \epsilon$, where $\Delta \epsilon=m v \Delta v+m \Delta v^{2} / 2$, where $\Delta v$ is a random variable with equal probability of being positive or negative. The magnitude of $\Delta v$ is a parameter that depends on the wave amplitude. The excursions in $\mu$ and $P_{\phi}$ follow from Eqs (2) and (4).

The IBW wave is modelled as described in the text accompanying Fig. 1. The mode that we call 'TAElike' has certain characteristics in common with a TAE mode, but we do not attempt to be precise either with respect to the mode or the wave-particle interaction. Rather, key features of the interaction with the TAE mode, or other similar modes, are retained. Specifically, we imagine this mode to be sufficiently below the ion cyclotron frequency that the invariance of $\mu$ is not broken, i.e. the only resonances encountered are Landau resonances, $n=0$, in Eq. (3). Hence, in interacting with the wave, the $\alpha$ particle experiences kicks in $\epsilon-\mu-P_{\phi}$ space with $\mu$ conserved.

While the diffusion path for the TAE-like mode is exact for any wave with frequency $\omega$ and toroidal mode number $n_{\phi}$, the probability of a kick and the possible correlation between kicks depend on more precise wave models than are employed here. Our assumption, which may not model precisely any low frequency mode, is that the mode is global, but a significant wave-particle interaction occurs only where there is a local resonance. This model TAE-like mode interacts with $\alpha$ particles in such a way as to retain certain key features of the TAE mode interaction with $\alpha$ particles. First, $\alpha$ particles are expelled by the model TAElike mode with the correct energy, since the orbits are precisely constrained by Eq. (2) and not affected by the details of the model. Second, although there is no 
threshold for stochastic interaction in our model mode, there is a threshold for expelling $\alpha$ particles, since there must be overlap of the TAE modes to deliver an $\alpha$ particle to the boundary. This can be modelled through a resonance broadening - at very small wave amplitudes, the wave-particle resonances given by Eq. (3) must be satisfied exactly, while for larger amplitudes this condition acquires a finite width, the extent of which depends on the wave amplitude. In practice, only a finite width resonance is effective at expelling the $\alpha$ particles. Thus, the model TAE-like mode at a given $n_{\phi}$ and at a given $\omega$ expels $\alpha$ particles with the same exit energy as a TAE mode with the same wave characteristics, and, like the TAE mode, expels these $\alpha$ particles only if a threshold condition is met.

We may recall Fig. 1, where the interaction of an $\alpha$ particle with only an IBW wave is shown. The succession of orbits depicted assumes that between orbits the $\alpha$ particle has lost energy to the wave according to Eqs (2) and (4). The result is that the $\alpha$ particle cannot reach the plasma periphery by interacting only with this wave. Now consider instead the interaction of this $\alpha$ particle with only a TAE-like mode; in Fig. 3(a) such an interaction is depicted, where, upon repeated interactions, the $\alpha$ particle orbit can explore the set of orbits shown. The $\alpha$ particle begins near the plasma centre at $3.5 \mathrm{MeV}$; it can then explore the outer orbits, with the outermost orbit at $2.7 \mathrm{MeV}$. None of these orbits, however, intersects the plasma boundary, so the $\alpha$ particle remains bracketed in both position and energy.

The simultaneous presence of both of these waves, however, leads to different behaviours. In Fig. 3(b), we depict the $\alpha$ particle succeeding in reaching the plasma periphery if both waves are present and if the $\alpha$ particle always loses energy upon resonating with either wave. In this case, where all kicks are to lower energy, the $\alpha$ particle loses $2.3 \mathrm{MeV}$ to the waves before exiting at the plasma periphery.

In the case that only one wave is present, the complete set of orbits accessible to the $\alpha$ particle is a line in $\epsilon-\mu-P_{\phi}$ space and can be generated simply: generate one set of orbits by assuming that between orbits the $\alpha$ particle only loses energy and generate a second set of orbits by assuming that between orbits the $\alpha$ particle only gains energy; the accessible space is the union of these two sets. Thus, in Fig. 2 and in Fig. 3(a), it is possible to conclude that if the $\alpha$ particle loses energy it must be found somewhere in the set of orbits depicted. In the case of two waves, however, the accessible region is a surface in $\epsilon-\mu-P_{\phi}$ space,
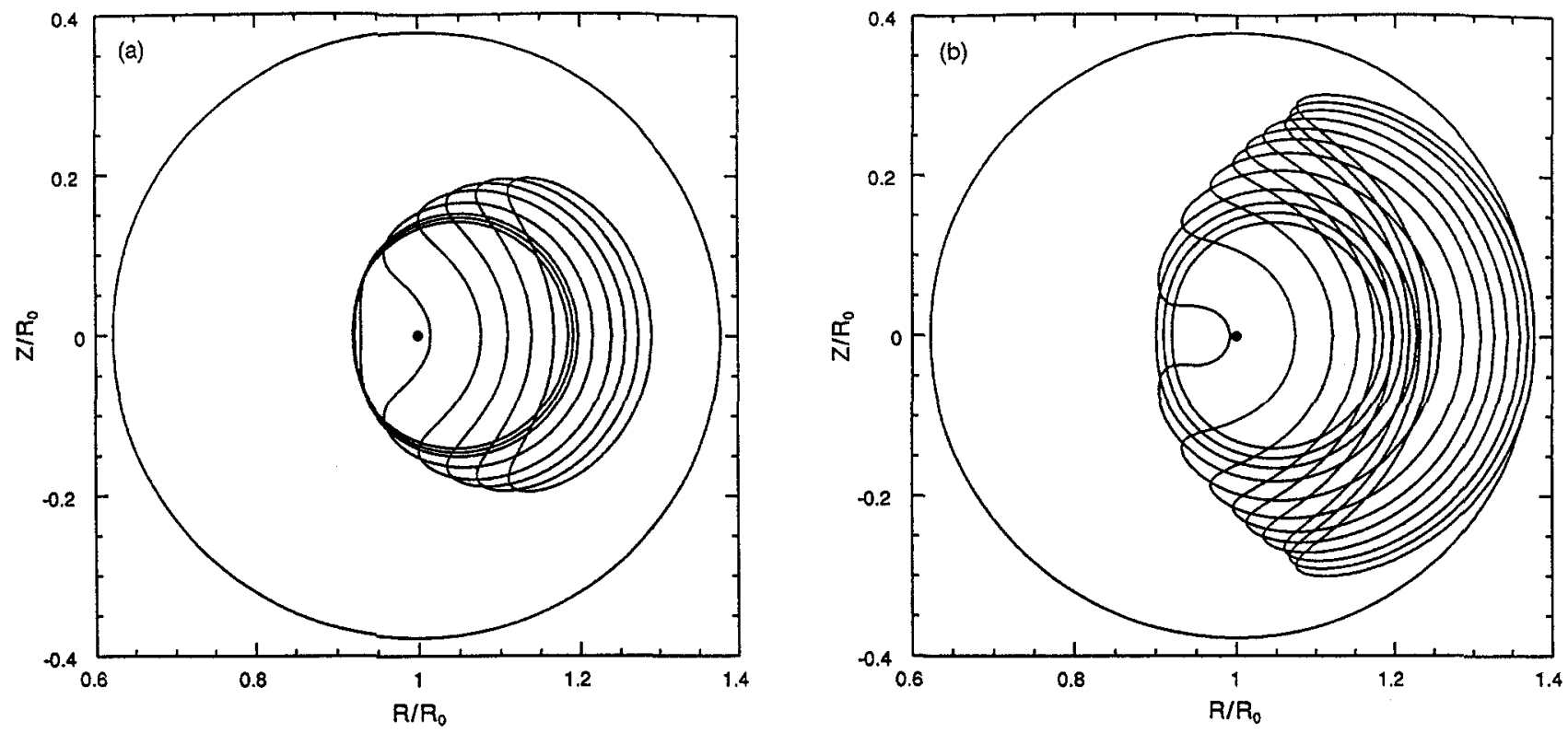

FIG. 3. (a) Poloidal cross-section of co-going $\alpha$ particle interacting solely with a TAE-like mode. Parameters as in Fig. 1. If the particle were to lose $0.8 \mathrm{MeV}$ under interaction with this wave it would move from the central orbit to the outermost trapped orbit. The particle cannot proceed further because it would no longer be resonant with the wave. (b) Same as Fig. 3(a) except that the $\alpha$ particles interact with both the MCIBW and a TAE-like mode, exiting at the periphery at $1.2 \mathrm{MeV}$. 


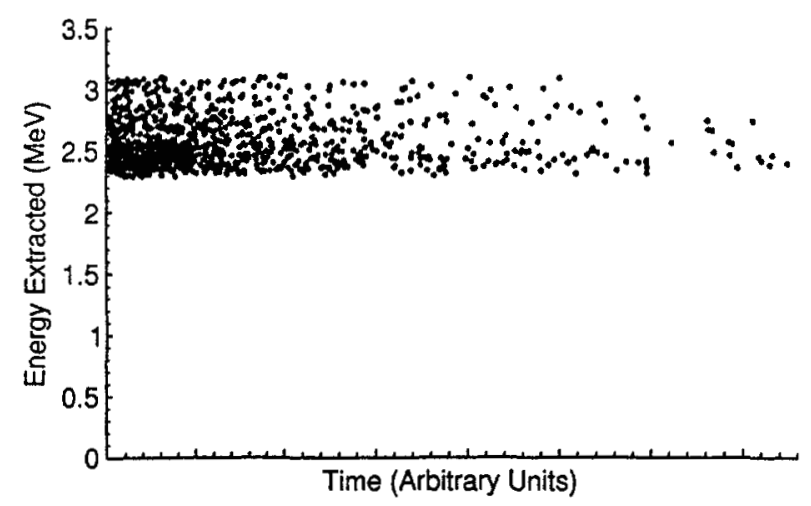

FIG. 4. Energy extracted versus time to extract for particle in Fig. $3(b)$.

and Fig. 3(b) represents only one possible realization. Although in the realization depicted the $\alpha$ particle did reach the periphery while losing energy, there is no guarantee that this need occur most of the time for random kicks. What has been shown, however, is that there is at least a realizable pathway to the periphery and to lower energy.

It is clear, in addition, that if there is one sequence of kicks that results in the $\alpha$ particle exiting the plasma, then, if the $\alpha$ particle receives random kicks from the wave, it must eventually exit the plasma.

In Fig. 4, we show the exit energy as a function of exit time for the same $\alpha$ particle subjected to random sequences of kicks. Interestingly, the sequence that leads to the most direct exit, namely the sequence depicted in Fig. 3(b) in which energy is always extracted, does not lead to the largest extracted energy at the particle exit. Note too that, although the $\alpha$ particle can wander around for long times before exiting, nonetheless substantial energy is still extracted. In Fig. 5, we integrate over the exit times to show the distribution of exit energies for random sequences of kicks.

The wave-particle interaction depicted in Figs $3-5$ is not necessarily optimized for energy extraction. In Fig. 6, by modifying somewhat the wave characteristics, we demonstrate how substantially all of the energy might be extracted from the same $\alpha$ particle. Here, there is substantially less overlap in space between the IBW waves and the TAE-like mode, so that the $\alpha$ particle first interacts with the TAE-like mode, essentially diffusing in one dimension, and then subsequently interacts with the IBW wave, also essentially diffusing on a line. In this case, as in the previous one, the presence of both waves is required to

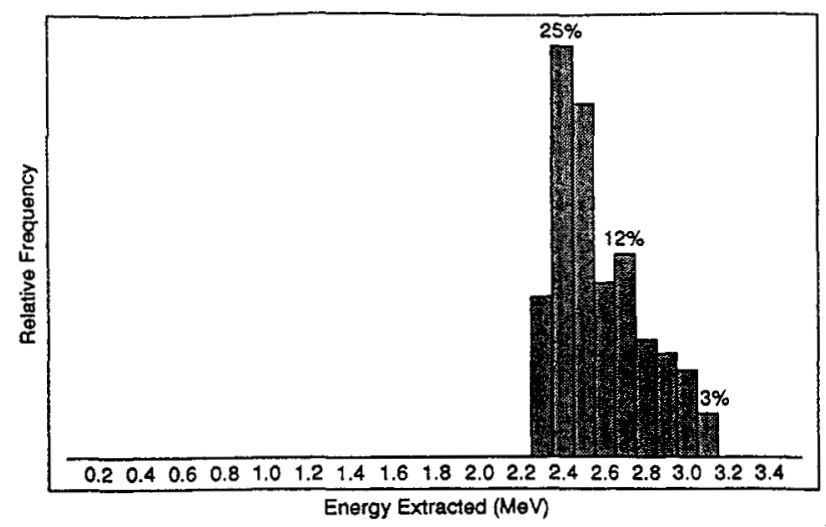

FIG. 5. Distribution of energy extracted in Fig. 4.

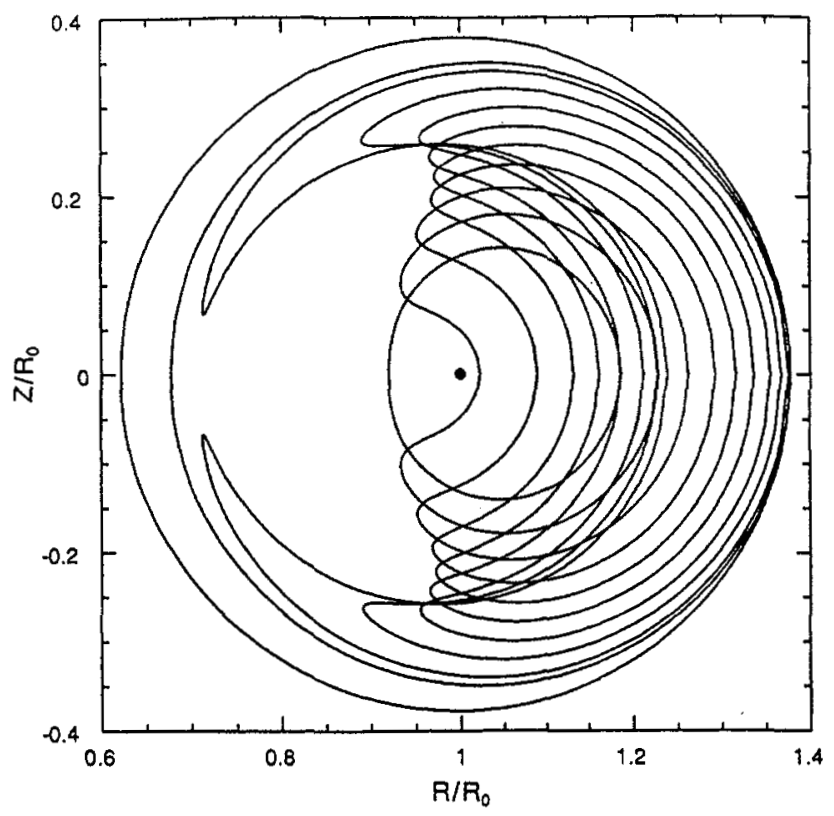

FIG. 6. Poloidal cross-section for co-going $\alpha$ particle as in Fig. $3(b)$ interacting with different $M C I B W$ and TAE waves and exiting at the periphery at $0.3 \mathrm{MeV}$.

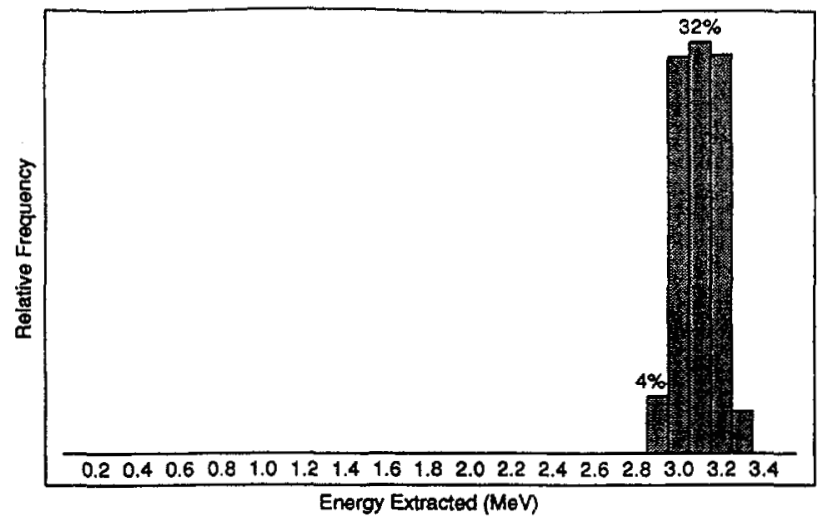

FIG. \%. Distribution of energy extracted, under interaction with waves as given in Fig. 6 . 
expel the $\alpha$ particle. In Fig. 7, we show the distribution of exit energies for random sequences of kicks and for waves as given in Fig. 6 . Note that the $\alpha$ particle tends to exit in a band of energies even narrower than in the first case. As demonstrated by these examples, the presence of more than one wave creates possibilities in expelling $\alpha$ particles that would not be present with only either wave. At the same time, the relaxation of the constraint that the $\alpha$ particle diffusion occurs only on a line is, at least in these examples, not significant enough to affect the tendency of the $\alpha$ particles to be cooled by the wave.

\section{CONCLUSIONS}

In developing a scenario for channelling $\alpha$ particle power, the first step must be to imagine how $\alpha$ particles might diffuse to the periphery while releasing nearly all of their energy. This paper offers an explanation of how this might come about. The interaction of several waves with an energetic ion population can lead to behaviour radically different from what can be accomplished with one wave only.

Of course, several significant caveats must be offered. First, the waves here are only coarsely modelled. The models employed here of the IBW wave and the TAE mode retain only the main features of these waves, not the details of their propagation and damping. Hence, the waves as described here are not expected to be realizable in detail. In addition, the wave-particle interaction is also only coarsely modelled. In particular, the use of a local resonance condition is probably a good model for the IBW wave, but a poor model for at least the TAE mode interaction with trapped $\alpha$ particles. Further, while we have reason to believe that energy extracted by either the TAE mode or the IBW wave could be damped on ions, this has not been shown for the model waves employed here.

Even if the IBW wave and the TAE mode could be excited in the plasma to accomplish exactly what has been illustrated in this paper, it must be realized that what may be the perfect combination of waves for some $\alpha$ particles may not be effective at all in channelling energy from other $\alpha$ particles. Thus, the next step must be to show that many $\alpha$ particles can be controlled effectively by the same set of waves. Also, the time-scale for producing the channelling effect must be shorter than the $\alpha$ particle slowing down time. In the simulations described here, this is assumed to be the case. The wave amplitudes required to produce this effect are probably realizable in smaller tokamak reactors if substantially all the $\alpha$ particle power is chan- nelled into the waves, but, until a better model of the waves is employed, this is by no means certain. Finally, the concentric circular flux surfaces used here are usefully simple, but neither an optimal nor even a realizable magnetic geometry for a tokamak reactor.

Nonetheless, as a first step to the development of the channelling scenario, this paper does demonstrate possibilities inherent in injecting several different kinds of waves. The combination of waves put forth here, one in the ion cyclotron range of frequencies or of higher frequency to break the $\mu$ invariant, and one lower frequency wave to move $\alpha$ particles large distances with relatively little energy exchange, appears to work well. The exploration of TAE-like modes is already quite developed, because of the potentially deleterious effects associated with the ejection of energetic $\alpha$ particles by these waves. Now there may be a further impetus, associated with possibly advantageous effects, for studying and controlling these modes.

\section{ACKNOWLEDGEMENTS}

This work was supported by the United States Department of Energy under contract number DEAC02-76-CHO3073. One of the authors (MCH) acknowledges the support of the Fannie and John Hertz Foundation.

\section{REFERENCES}

[1] FISCH, N.J., RAX, J.M., Phys. Rev. Lett. 69 (1992) 612.

[2] FISCH, N.J., RAX, J.M., in Plasma Physics and Controlled Nuclear Fusion Research 1992 (Proc. 14th Int. Conf. Würzburg, 1992), Vol. 1, IAEA, Vienna (1993) 769.

[3] CLARKE, J.F., Nucl. Fusion 20 (1980) 563.

[4] FISCH, N.J., HERRMANN, M.C., Nucl. Fusion 34 (1994) 1541.

[5] SIGMAR, D.J., in Physics of Plasmas Close to Thermonuclear Conditions (Proc. Course Varenna, 1979), Vol. 1, CEC, Brussels (1980) 271.

[6] SUTTON, W.R., III, et al., Fusion Technol. 7 (1985) 374.

[7] CHEN, K.R., Phys. Rev. Lett. 72 (1994) 3534.

[8] KRLÍN, L., PAVLO, P., Nucl. Fusion 34 (1994) 1517.

[9] KUPFER, K., et al., Nucl. Fusion 35 (1995) 163.

[10] VALEO, E.J., FISCH, N.J., Phys. Rev. Lett. 73 (1994) 3536.

[11] FISCH, N.J., Phys. Plasmas 2 (1995) 2375.

[12] ROME, J.A., PENG, Y-K.M., Nucl. Fusion 19 (1979) 1193.

[13] HSU, C.T., SIGMAR, D.T., Phys. Fluids B 4 (1992) 1492.

[14] MYNICK, H.E., Phys. Fluids 5 (1993) 1471.

[15] MYNICK, H.E., POMPHREY, N., Nucl. Fusion 34 (1994) 1277.

[16] BERNSTEIN, I.B., Phys. Rev. 109 (1958) 10.

[17] PERKINS, F.W., Nucl. Fusion 17 (1977) 1197. 
[18] ONO, M., Phys. Fluids B 5 (1993) 241.

[19] MAJESKI, R., et al., Phys. Rev. Lett. 73 (1994) 2204.

[20] DARROW, D.S, et al., Enhanced Loss of Fast Ions During Mode Conversion Ion Bernstein Wave Heating in TFTR, (in preparation).

[21] CHENG, C.Z., et al., Ann. Phys. (N.Y.) 161 (1985) 21.

[22] CHENG, C.Z., CHANCE, M.S., Phys. Fluids 29 (1986) 3695 .
[23] BERK, H.L., et al., Phys. Rev. Lett. 68 (1992) 3563.

[24] FU, G.Y., VAN DAM, J.W., Phys. Fluids B 1 (1989) 1949.

[25] BERK, H.L., BREIZMAN, B.N., Phys. Fluids B 2 (1990) 2246.

[26] SIGMAR, D.T., et al., Phys. Fluids B 4 (1992) 1506.

[27] HUYSMANS, G.T.A., et al., Phys. Plasmas 2 (1995) 1605.

(Manuscript received 4 July 1995

Manuscript accepted 20 July 1995) 